CLAWAR 2018: 21st International Conference on Climbing and

Walking Robots and the Support Technologies for Mobile Machines,

Panama City, Panama, 10-12 September 2018

\title{
HUMANOID ROBOT: \\ A REVIEW OF OUR RESEARCH WORKS
}

\author{
XIE MING \\ Nanyang Technological University \\ 50 Nanyang Avenue, Singapore 639798
}

Since 1996, we have embarked into the journey of developing humanoid robots at Nanyang Technological University, Singapore. We have ventured into the various technical aspects of humanoid robot development. In particular, we have placed special emphasis on mechatronics design of humanoid robots, planning and control of biped walking, hand-eye coordination for humanoid robots, cognitive vision for humanoid robots, and cognitive speech for humanoid robots. Since 2006, several teams in Singapore have received a substantial amount of research grants and have developed together two full prototypes of humanoid robots, which are about 1.8 meters in height and weigh about $80 \mathrm{~kg}$ each. In addition, each humanoid robot has 42 degrees of freedom with independent actuations. In this plenary talk, I will share some findings and results related to the R\&D works of humanoid robot in Singapore. 